Association for Information Systems AIS Electronic Library (AISeL)

Wirtschaftsinformatik Proceedings 2005

Wirtschaftsinformatik

February 2005

\title{
Information Feedback in Iterative Combinatorial Auctions
}

\author{
Alexander Pikovsky \\ Technische Universität München, Germany \\ Martin Bichler \\ Technische Universität München, Germany
}

Follow this and additional works at: http://aisel.aisnet.org/wi2005

\section{Recommended Citation}

Pikovsky, Alexander and Bichler, Martin, "Information Feedback in Iterative Combinatorial Auctions" (2005). Wirtschaftsinformatik Proceedings 2005. 18.

http://aisel.aisnet.org/wi2005/18

This material is brought to you by the Wirtschaftsinformatik at AIS Electronic Library (AISeL). It has been accepted for inclusion in Wirtschaftsinformatik Proceedings 2005 by an authorized administrator of AIS Electronic Library (AISeL). For more information, please contact elibrary@aisnet.org. 
In: Ferstl, Otto K, u.a. (Hg) 2005. Wirtschaftsinformatik 2005: eEconomy, eGovernment, eSociety; 7. Internationale Tagung Wirtschaftsinformatik 2005. Heidelberg: Physica-Verlag

ISBN: 3-7908-1574-8

(C) Physica-Verlag Heidelberg 2005 


\title{
Information Feedback in Iterative Combinatorial Auctions
}

\author{
Alexander Pikovsky, Martin Bichler \\ Technische Universität München, Germany
}

\begin{abstract}
Auctions have been getting increasing attention in computer science and economics, as they provide an efficient solution to resource allocation problems with self-interested agents. E-Commerce and finance have emerged as some of their largest application fields. The need for new auction mechanisms that allow complex bids such as bundle or multi-attribute bids has been raised in many situations. Beyond strategic problems, the design of these advanced auction formats exhibits hard computational problems. Pricing is one of the major challenges in designing iterative combinatorial auctions. The presence of bundle bids implies the existence of cases with no linear prices that support competitive equilibrium. This paper introduces a framework of pricing concepts and discusses recent implementations.
\end{abstract}

Keywords: Combinatorial auction, multidimensional auction, pricing, primal-dual algorithm

\section{Introduction}

Auctions have been found to be efficient economic mechanisms for resource allocation in distributed environments with self-interested agents [Klem99]. They have found numerous applications in finance and e-commerce, and provide a promising technique for coordination in many computational environments such as agent-based systems. The typical auction consists of the bid submission, bid evaluation (a.k.a. winner determination, market clearing, or resource allocation), and the calculation of settlement prices, followed by some feedback to the bidders in an iterative, or open-cry auction (see Figure 1). Auctions close either at a fixed point in time or according to a certain stopping rule (e.g. no new bids were submitted). The competitive process of auctions serves to aggregate the scattered information about bidders' valuations and to dynamically set prices of a trade.

A fundamental shortcoming of traditional auction mechanisms is their inability to allow for complex bid structures which exploit complementarities and economies of scale in cost structures of bidders. As many organizations have begun to realize the efficacy of auctions, interest has emerged to extend basic auction types to sup- 
port negotiations beyond price, and communicate bids with a more complex set of preferences. For example, procurement of direct inputs is usually very large and requires the use of special price negotiation schemes that incorporate appropriate business practices.

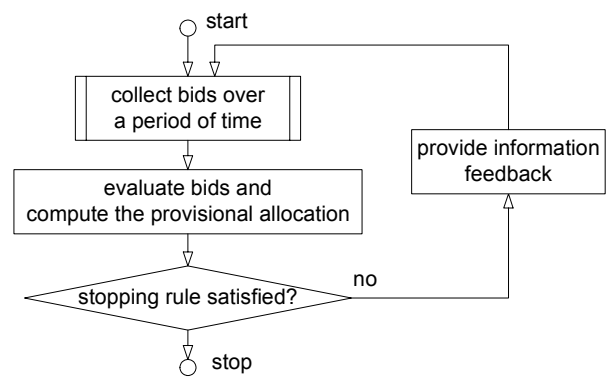

Figure 1: Process of an Iterative Auction

Typically, bids in these settings have the following properties:

- large transaction volume, volume discounts provided by suppliers

- all-or-nothing bids on a set of items with a special discounted price

- multiple, non-price attributes of a good can be traded off against the price

Volume discount auctions facilitate negotiations on large quantities of goods [DaKa00], combinatorial auctions allow bids on packages [RP98, Nis00], whereas multiattribute auctions facilitate negotiation on multiple attributes [Bich01]. These "multidimensional" auctions have performed well in the lab, but also in a number of real-world implementations (see [ $\left.\mathrm{Cra}^{+} 04\right]$ for combinatorial auctions).

\begin{tabular}{|l|c|c|c|c|}
\hline & \multicolumn{4}{|c|}{ Suppliers } \\
\hline Items & S1 & S2 & S3 & S4 \\
\hline 10 HD A 10GB & 1 & 0 & 1 & 1 \\
\hline 20 HD B 40GB & 0 & 1 & 1 & 1 \\
\hline 20 HD C 60GB & 1 & 1 & 1 & 0 \\
\hline Bid Price & $€ 4000$ & $€ 5800$ & $€ 6700$ & $€ 3500$ \\
\hline
\end{tabular}

Table 1: Combinatorial Bid Example

Table 1 illustrates an example with a combinatorial reverse auction for computer hard drives with 4 suppliers. Each supplier provides a bundled "all-or-nothing" bid. Notice that as the number of items increases the number of bids can grow exponentially. After collecting bids, the buyer needs to identify the set of bids that minimizes total procurement cost subject to business rules such as limits on the number of winning bidders, or the amounts purchased from certain bidders or groups of bidders. Identifying such bid set is a hard optimization problem. Therefore, winner determination problem is central to most multidimensional auctions. 
Due to several reasons (see section 2.3) iterative auction formats have emerged as the predominant form of combinatorial auctions $(C A)$ in practice. However, designing iterative combinatorial auctions (ICA) has turned out to be a challenging task. One of the main problems in ICA is the calculation of feedback prices. Determining appropriate prices for bundle bids is important for market designers who are interested in market efficiency, and for bidders who need information on how to improve their bids in order to become a winner.

In this paper we outline a framework of different pricing concepts and auction formats for ICA and discuss recent implementation approaches. In section 2 we give an overview of some combinatorial auction design issues and formulate the winner determination problem. In section 3 we introduce different pricing schemes used in ICA, discuss their impact on the auction efficiency and describe the general model of primal-dual auctions. In section 4 we provide a classification of the ICA, give an overview of several selected implementations and discuss them with respect to the concepts defined in the previous sections. Section 5 concludes with some summarizing remarks.

\section{Combinatorial Auctions}

Combinatorial auctions $(C A)$ can be described as decentralized resource allocation mechanisms for environments where multiple bidders have super- or subadditive utility functions. The task of the auction mechanism is to allocate these resources in an "optimal" way.

\subsection{Auction Design Goals}

One goal in economic theory is the allocative efficiency, in which the auction mechanism maximizes the total payoff across all agents. Another goal is the revenue maximization, in which the auction maximizes the payoff to a particular participant, usually the auctioneer. The utility of the bidders for the various bundles of resources is private information and not known to the auctioneer. Auction design can be described as a set of rules, which motivate the bidders to reveal their true valuations to the extent that makes it possible to solve for the optimal allocation. Overall, the auction rules can be categorized as follows:

- the auction protocol, i.e. the sequence, syntax and semantics of messages exchanged throughout the auction.

- the allocation rules, which include the overall objective of the allocation (i.e. efficiency vs. payoff maximization), as well as additional allocation constraints. 
- the payment rules, which determine the payment from or to the winner(s).

As in traditional auction design, the allocation rules, the auction protocol and the payment rules impact the bidders' strategies. Auction designers try to construct incentive-compatible auctions: auctions in which bidders report truthful information about their preferences, and they do so out of their own self-interest. Strategy proof mechanisms are even stronger in that truthful bidding has to be a dominant strategy. The second-price sealed-bid (Vickrey) auctions or, more generally, Vickrey Clarke Groves (VCG) mechanisms are an example of strategy proof mechanisms. Bidding strategies and desirable economic properties of auctions have been analyzed extensively in the context of mechanism design theory [Mas ${ }^{+}$95].

Computational complexity has not been a major concern in traditional mechanism design [One $\left.{ }^{+} 04\right]$. Indivisibilities (also called non-convexities) as they occur in multidimensional auctions (due to bundle bids or economies of scale) typically lead to computationally hard problems. For example, the winner determination problem in combinatorial auctions belongs to the class of NP-hard problems (see section 2.2). Much of the early literature on combinatorial auctions has focused on this very aspect. However, also calculating feedback prices becomes a computationally difficult task (see section 3.2).

\subsection{The Winner Determination Problem}

The winner determination problem (WD, a.k.a. Combinatorial Auction Problem, CAP) can be modeled as an instance of the weighted set packing problem which is known to be NP-complete, and has a straight-forward IP formulation.

$$
\begin{aligned}
& \max _{x_{i}(S)} \sum_{S} \sum_{i} x_{i}(S) v_{i}(S) \\
& \text { s.t. } \\
& \sum_{S} x_{i}(S) \leq 1, \quad \forall i \in I \\
& \sum_{S: j \in S} \sum_{i} x_{i}(S) \leq 1, \quad \forall j \in G \\
& \quad x_{i}(S) \in\{0,1\}, \forall i, S
\end{aligned}
$$

The objective function maximizes overall revenue with $S$ being the item bundles, $v_{i}(S)$ being the valuation or price bid for bundle $S$ by the bidder $i$, and $x_{i}(S)$ being a binary decision variable. The first set of constraints guarantees that any bidder can win at most one bundle ${ }^{1}$. The second set of constraints ensures that no item is sold

1 This is called XOR-bidding. The alternative is the OR-bidding, where one bidder can win multiple bundles. XOR-bidding is more flexible since it avoids the exposure problem (see section 2.3) still allowing bids on all possible bundles. 
more than once with $G$ being the set of items to be sold. The reverse auction case can be modeled as a set covering or set partitioning problem, respectively.

Another combinatorial auction design aspect is additional side constraints, which can affect the running time (see [ $\left.\mathrm{Kal}^{+} 04\right]$ ). For example, in a reverse auction, buyers want to make sure that the entire supply is not purchased from too few suppliers, since this creates a high exposure if some of them are not able to deliver on their promise. Another common constraint is volume-based budget limits, which are often placed as an upper total volume limit of the transaction with a particular bidder.

Integer programming techniques can be used to handle winner determination in combinatorial auctions with a "small enough" number of items. On the other hand, various heuristics and approximation algorithms are likely to produce solutions which, in most cases, are optimal or close to optimal. However, suboptimality may not be adequate, if a market designer aims for economic efficiency. Rothkopf et al. ([RoPe98]) discuss limiting biddable combinations, which can make the winner determination problem tractable. A survey by de Vries and Vohra ([VrVo03]) addresses the literature of the last few years on algorithmic approaches. Similar computational problems can be found in volume discount auctions ([DaKa00]) and multi-attribute auctions ([BiKa04]).

\subsection{Combinatorial Auction Formats}

Revenue comparisons among auction mechanisms and the analysis of equilibrium bidding strategies are at the core of economic auction theory ([MM87, Wo196]). Currently, there is hardly any game-theoretical analysis of combinatorial auctions ([KR96, Lev97]). Nevertheless, classic auction theory can serve as a guideline. The first-price sealed-bid auction has been used as a model for some combinatorial auctions in practice ([ElKe02]), however they exhibit high strategic complexity for the bidder. Alternatively, VCG mechanisms were proposed, that make it a dominant strategy for bidders to bid their true valuations. They do this by refunding to the bidders the increase in overall revenue caused by their bids. With the bidders' truthful valuations, the bid taker can achieve allocative efficiency.

Unfortunately, VCG mechanisms are impractical and hardly ever used. First of all, bidders need to reveal their entire utility function, i.e. to submit bids for all $2^{n}$ possible bundles. This leads to a high valuation complexity for the bidders, but also to a large input size to the WD problem. In addition, the determination of the Vickrey payments itself becomes a computationally hard problem. Another problem is the need of a trusted auctioneer. The winner in a second price auction has to doubt, whether the auctioneers payment request is actually the second-highest price, and to worry whether the auctioneer reveals their valuations to other auction participants. Cryptographic methods have been proposed to solve this problem ([Bran03]). 
Many researchers consider iterative auctions as an alternative. Iterative formats such as the English auction are very popular in electronic commerce applications. They allow bidders to learn about their competitors' bids, which is an important aspect if bidders' valuations are affiliated ([Milg87]). In iterative auctions, bidders do not have to submit bids on all possible bundles at once, but can bid only on a small number of bundles in each round.

Unfortunately, designing ICA leads to a number of other difficulties. For example, the well-known threshold problem refers to the difficulty that multiple bidders desiring small bundles that constitute a large bundle may have in outbidding a single bid for the large bundle. The exposure problem considers the risk of winning items at prices above the valuations, which usually happens if a bidder with a superadditive valuation of bundle wins only a part of this bundle. Though the exposure problem is usually typical only for pseudo-combinatorial auctions (see section 4), it can also become relevant for combinatorial auctions in case of OR-bidding. Avoiding or resolving ties can become a problem, because allocations can be composed of multiple winners. In addition, the amount of communication between the bidders and the auctioneer can become quite high ([NiSe02]). This is also referred to as communicative complexity. However, the most fundamental problem in the ICA design is determining feedback prices in each iteration.

\section{Theory of Iterative Combinatorial Auctions}

The key challenge in the ICA design is to provide information feedback to the bidders after each iteration. Pricing (assigning prices to items and/or item bundles) was adopted as the most intuitive mechanism of providing feedback, especially for ICA with auctioneer-side allocation (see Figure 3, section 4).

In contrast to the single-item single-unit auctions, pricing is not trivial for ICA. The main difference is the lack of the natural single-item prices. With bundle bids setting independent prices for individual items is not obvious and often even impossible. In the following sections we introduce 3 different pricing schemes and discuss their impact on the auction outcome.

\subsection{Pricing Schemes}

Definition 1. A set of prices $p_{i}(S), i \in \mathrm{I}, S \subseteq \Gamma$ is called:

- $\quad$ linear (or additive), if $\forall i, S: p_{i}(S)=\sum_{j \in S} p_{i}(j)$

- anonymous, if $\forall k, l, S: p_{k}(S)=p_{l}(S)$ 
In other words, prices are linear if the price of a bundle is equal to the sum of the prices of its items, and anonymous if the prices of the same bundle are equal for every bidder. The non-anonymous prices are also called discriminatory prices.

The following 3 pricing schemes ${ }^{2}$ can be derived using the above definitions:

1. linear anonymous prices

2. non-linear anonymous prices

3. non-linear discriminatory prices

The first pricing scheme is obviously the simplest one. Linear anonymous prices are easily understandable and usually considered fair by the bidders. The communication costs are also minimized, because the amount of information to be transferred is linear in the number of items. The second pricing scheme introduces the non-linearity property, which is often necessary to express strong super- or subadditivity in the bidder valuations. Unfortunately, non-linear prices are often considered too complex and the communication costs also increase. If even non-linear anonymous prices are not sufficient to lead the auction to competitive equilibrium, the third pricing scheme can be used. ${ }^{3}$ However, discriminatory pricing introduces additional complexity and is often considered unfair by the bidders.

In the following sections we introduce the notions of the prices compatible with an allocation and the competitive equilibrium prices. We also summarize important theoretical results regarding the existence of these kinds of prices and the impact of the pricing schemes.

\subsubsection{Compatible Prices}

Definition 2. A set of prices $p_{i}(S)$ is called compatible with the allocation $x_{i}(S)$ and valuations $v_{i}(S)$, if

$$
\forall i, S: x_{i}(S)=0 \Leftrightarrow p_{i}(S)>v_{i}(S) \text { and } x_{i}(S)=1 \Leftrightarrow p_{i}(S) \leq v_{i}(S)
$$

The interpretation is quite intuitive: the set of prices is compatible with the given allocation at the given valuations if and only if all winning bids are higher than or equal to the prices and all loosing bids are lower than the prices (assuming the bidders bid at their valuations). This is best visualized by the following example.

Example 1. Compatible prices.

There are 2 bidders and 2 items, the bidder valuations (bids) are given by the following table (bids belonging to the optimal allocation are marked with a star):

To our knowledge linear discriminatory prices have been hardly considered in the context of combinatorial auctions.

3 Due to [BO02] non-linear discriminatory competitive equilibrium prices do always exist and support the efficient allocation. 


\begin{tabular}{|c|c|c|c|}
\hline & A & B & AB \\
\hline Bidder 1 & 2 & 3 & 5 \\
\hline Bidder 2 & 1 & 4 & $7^{*}$ \\
\hline
\end{tabular}

Table 2: Compatible prices example 1

Consider the optimal allocation $x_{2}(A B)=1$ with the total revenue of 7 first. We can easily construct non-linear compatible prices by setting for instance $p(A)=100, p(B)=100, p(A B)=7$. On the contrary, constructing linear compatible prices is not a trivial task. The price set $p(A)=2, p(B)=4, p(A B)=6$ is not compatible, because the bidder 1 would get the item $A$ and the bidder 2 would also get the item $B$ (the compatibility conditions are violated for $\{1, A\}$ and $\{2, B\}$ ). The price set $p(A)=3, p(B)=5, p(A B)=8$ is not compatible, because the bidder 2 would not get the bundle $A B$ (the compatibility conditions are violated for $\{2, A B\})$. The compatible price set can be found for instance at $p(A)=2.5$, $p(B)=4.5, p(A B)=7$.

Now consider a non-optimal allocation $x_{2}(B)=1$ with the total revenue of 4 , which does not allocate the item $A$ at all. In this case (even linear) compatible prices can also be constructed by setting the price of the item $A$ high enough, for example $p(A)=100, p(B)=4, p(A B)=104$. Notice also that if an allocation assigns any bundle to a non-highest bidder of this bundle, no compatible prices can be constructed at all.

Compatible prices explain the winners why they won and the losers, why they lost. In fact, informing the bidders about the allocation $x_{i}(S)$ is superfluous, if compatible prices are communicated. However, the above example shows that not every set of compatible prices provides the bidder with a meaningful information for improving her bids in the next auction iteration. Another important observation is the fact that linear compatible prices are harder and often even impossible (see Example 3 in the following subsection) to construct, when the bidder valuations are super- or subadditive.

\subsubsection{Competitive Equilibrium Prices}

Definition 3. A set of prices $p_{i}(S)$ is in competitive equilibrium $(C E)^{4}$ with the allocation $x_{i}(S)$ and valuations $v_{i}(S)$, if

1. The prices pi(S) are compatible with the allocation $x i(S)$ and valuations vi(S)

2. Given the prices pi(S), there exist no allocation with larger total revenue than the revenue of the allocation $\mathrm{xi}(\mathrm{S})$

4 This definition is due to [Par01]. Sometimes CE prices are defined only by the condition 1, but the existence of CE prices do not imply the optimality of the allocation in this case. 
The idea behind this concept is to define prices characterizing the optimal allocation. The prices may not be too low to violate the compatibility condition (1), but they may not be too high either to violate the condition 2. In general, one can show that the existence of CE prices implies optimality of the allocation and that the opposite is also true in case of non-linear discriminatory prices:

Theorem 1. Following statements are true:

1. If an allocation $x i(S)$ and prices pi(S) are in competitive equilibrium for the given valuations vi(S), this allocation is the optimal allocation.

2. For the optimal allocation $x i(S)$ there always exist discriminatory non-linear competitive equilibrium prices pi(S). This is not always true for linear and anonymous non-linear prices.

We will outline the proof idea of this theorem in the section 3.2.2. ${ }^{5}$

The following examples provide a better understanding of the CE prices. The Example 2 continues the Example 1. It illustrates which price sets constructed in the Example 1 are $\mathrm{CE}$ and shows that no $\mathrm{CE}$ prices exist for the non-optimal allocation. The Example 3 and Example 4 demonstrate cases where no linear and even no anonymous non-linear $\mathrm{CE}$ prices exist for the optimal allocation.

Example 2. Competitive equilibrium prices.

For the optimal allocation $x_{2}(A B)=1$ with the total revenue of 7 in the Example 1 we constructed two compatible price sets, so only the competitive equilibrium condition 2 has to be verified. At the prices $p(A)=2.5, p(B)=4.5, p(A B)=7$ the most profitable possibilities are to sell the items either in a bundle for the price of 7 or separately for the total price of $2.5+4.5=7$. In both cases this is exactly the revenue of the considered allocation $x_{2}(A B)=1$, so the prices are in competitive equilibrium. In contrast, the prices $p(A)=100, p(B)=100, p(A B)=7$ are not $\mathrm{CE}$, because the allocation $x_{1}(A)=1, x_{2}(B)=1$ with the total revenue of $100+100=200$ would be better than the allocation $x_{2}(A B)=1$ at the current prices.

For the non-optimal allocation $x_{2}(B)=1$ with the total revenue of 4 the price set $p(A)=100, p(B)=4, p(A B)=104$ is not a competitive equilibrium either, because the auctioneer can get more revenue by selling the item $A$. Moreover, no CE prices exist for this allocation, since the price of the bundle $A B$ has to be larger than 7 to ensure compatibility, but in this case selling the bundle $A B$ would bring more revenue than the considered allocation $x_{2}(B)=1$.

Example 3. Linear CE prices do not always exist.

There are 3 bidders and 3 items, the bidder valuations (bids) are given by the following table:

5 For complete proof see [BO02] or [Par01]. 


\begin{tabular}{|l|l|l|l|l|l|l|l|}
\hline & A & B & C & AB & BC & AC & ABC \\
\hline Bidder 1 & 60 & 50 & 50 & $\mathbf{2 0 0 *}$ & 100 & 110 & 250 \\
\hline Bidder 2 & 50 & 60 & 50 & 110 & $\mathbf{2 0 0}$ & 100 & 255 \\
\hline Bidder 3 & 50 & 50 & $\mathbf{7 5 *}$ & 100 & 125 & $\mathbf{2 0 0}$ & 250 \\
\hline
\end{tabular}

Table 3: Competitive equilibrium prices example 3

The optimal allocation is $x_{1}(A B)=1, x_{3}(C)=1$ with the total revenue of 275 . To be compatible with this allocation, prices must satisfy the following inequalities:

$$
\begin{aligned}
& p(A)+p(B) \quad \leq 200 \quad p(A)+p(C) \quad>200 \\
& p(C) \leq 75 \quad p(B)+p(C) \quad>200 \\
& p(A)+p(B)+2 p(C) \leq 350 \quad p(A)+p(B)+2 p(C)>400
\end{aligned}
$$

which is a contradiction. This proves that no linear compatible prices (and so no linear CE prices) exist for the optimal allocation in this case. The reason is the strong superadditive bidder valuations of multiple item bundles.

Example 4. Anonymous non-linear CE prices do not always exist.

There are 2 bidders and 2 items, the bidder valuations (bids) are given by the following table:

\begin{tabular}{|l|l|l|l|}
\hline & A & B & AB \\
\hline Bidder 1 & 0 & 0 & $3^{*}$ \\
\hline Bidder 2 & 2 & 2 & 2 \\
\hline
\end{tabular}

Table 4: Competitive equilibrium example 4

The optimal allocation is $x_{l}(A B)=1$ with the total revenue of 3 . To be compatible with this allocation, the (anonymous) item prices $p(A)$ and $p(B)$ both have to be larger then 2. This implies that the auctioneer can get the total revenue of at least 4 by selling the items separately, which is larger then the total revenue of the considered allocation $x_{1}(A B)=1$. This proves that no anonymous CE prices exist for the optimal allocation in this case.

Note that for all considered examples we can easily construct discriminatory nonlinear CE prices for the optimal allocation. Generally, the more bundle valuations are super- or subadditive and the stronger these super- or subadditivities are, the harder it is to find linear or anonymous non-linear prices.

Note also that the competitive equilibrium conditions can be directly verified by the auctioneer if the agents follow the myopic best-response bidding strategy ${ }^{6}$. In

6 The myopic best-response bidding strategy or some similar concept is often assumed to simplify the auction analysis. In it each bidder maximizes her utility (value - price) by always biding for the most profitable bundle at the current prices. For details see [Par01]. 
fact, given an allocation and a price set, the auctioneer can verify the second CE condition without knowing bidder valuations. The first $\mathrm{CE}$ condition is satisfied (assuming myopic best-response bidding), if all current loosing bids are lower than the prices, all current winning bids are not lower than the prices, and no new bids are submitted in the next iteration.

The equivalence of the allocation efficiency and the existence of CE prices encourages designing an iterative auction, which ends up with CE prices. One important systematic approach to do that is the primal-dual auction algorithms.

\subsection{Primal-Dual Auction Algorithms}

The fundamental work of Bikhchandani and Ostroy [BiOs02] demonstrates a strong interrelationship between the iterative auctions and the primal-dual linear programming algorithms. A primal-dual linear programming algorithm can be interpreted as an auction where the dual variables represent item prices. The algorithm maintains a feasible allocation and a price set and terminates as the efficient allocation and competitive equilibrium prices are found. In this section we give an overview of the primal-dual algorithms in integer programming and their applications to the combinatorial auction design.

\subsubsection{LP Duality and Integer Programming - A Short Overview}

The primal-dual linear programming algorithms use the weak and strong duality theorems, namely the fact that for any pair of feasible primal and dual solutions the (primal) objective function value of the primal solution is never larger than the (dual) objective function value of the dual solution, and that these values are equal if and only if both solutions are optimal for the correspondent problems.

A primal-dual algorithm usually maintains a feasible dual solution and tries to compute a primal solution that is both feasible and satisfies the complementary slackness conditions. ${ }^{7}$ If such a solution is found, the algorithm terminates. Otherwise the dual solution is updated towards optimality and the algorithm continues with the next iteration. The progress of a primal-dual algorithm applied to a linear program is illustrated by the Figure 2 (a).

Unfortunately, primal-dual algorithms can not be directly applied to an integer program if the feasible region of its linear relaxation has fractional extreme points. ${ }^{8}$ In this case the optimal solution of the linear relaxation may be fractional. To illustrate the problem consider two different ways of applying the primal-dual

\footnotetext{
For an overview of the linear and integer programming see [NW88, PS98].

Several dual-based procedures for solving integer programs are known (see [dVV03, NW88, PS98]).
} 
approach to an integer program. If we apply it to the linear relaxation of the original problem we will end up with a fractional solution. Alternatively, we can re-

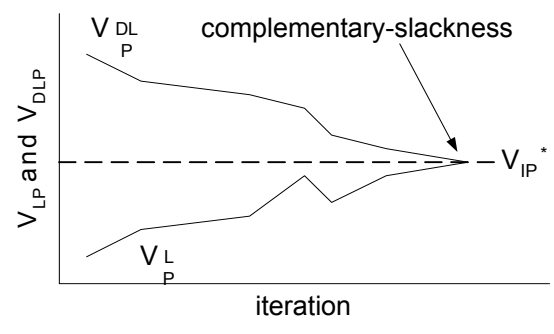

(a) LP or "strong" IP formulation

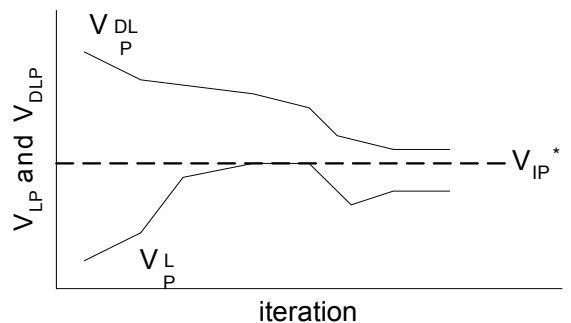

(b) "Weak" IP formulation

Figure 2: Progress of a primal-dual algorithm

strict all computed primal solutions to be integer. In this case, however, complementary-slackness conditions are never satisfied and the algorithm will never identify the optimal solution. Additional stopping rules can be introduced, but the optimality of the best found primal solution is not guaranteed. One possible scenario is shown in the Figure 2 (b).

Note that if the feasible region of the linear relaxation has only integer extreme points, the primal-dual algorithm will always find the optimal solution. The problem formulation is called "strong" or "exact" in this case, and it is called "weak" otherwise. A "weak" formulation of any combinatorial program can always be strengthened by adding additional inequalities (cutting planes) valid for all integer feasible solutions but cutting off some fractional feasible solutions of the relaxation. However, the facet structure of many relevant (usually NP-hard) integer problems is either unknown or too complex to be used in this way.

\subsubsection{Duality and Competitive Equilibrium Prices}

In this section we give a very short overview of the primal-dual framework for the combinatorial auction problem (for details see [Park01]).

Consider the linear relaxation of the CAP formulation introduced in the section 2.2, we also call it the level 1 formulation CAP1. The CAP1 can be shown to be weak. It can be strengthened by adding valid inequalities in a special way, which results in the level 2 formulation CAP2. The formulation CAP2 can be further strengthened by adding another family of valid inequalities resulting in the level 3 formulation CAP3, which can be shown to be strong (see [Park01]).

The manner in which the three levels of CAP are constructed results in the following important properties of the respective dual problems. The CAP1 dual variables can be interpreted as anonymous linear prices, the CAP2 dual variables - as anonymous non-linear prices and the CAP3 dual variables - as discriminatory 
non-linear prices. In all three cases it can be shown that the complementary slackness conditions are satisfied exactly when the current allocation (primal solution) and the prices (dual solution) are in competitive equilibrium (see [Park01]).

Now the proof idea of the Theorem 1 (section 3.1.2) can be sketched. If current prices are in competitive equilibrium with the provisional allocation, the complementary-slackness conditions are satisfied and the allocation is optimal. This proves the first part of the theorem. The second part is true since the CAP3 formulation is strong and, therefore, a primal-dual pair of integer solutions which satisfy the complementary-slackness conditions exist. The direct interconnection between auctions, pricing schemes and the duality theory motivates applying the primaldual approach to the iterative auction design.

\subsubsection{Primal-Dual Auction Algorithms}

Several auction formats based on the primal-dual approach have been proposed in the literature. Though these auctions differ in several aspects, the general scheme can be outlined as follows:

1. Choose minimal initial prices (usually set them to 0 ).

2. Announce current prices and collect bids. Bids have to be higher or equal than the prices.

3. Compute the current dual solution by interpreting the prices as dual variables. Try to find a feasible allocation (an integer primal solution) that satisfies the stopping rule. If such solution is found, stop and use it as the final allocation. Otherwise update prices appropriately and go back to 2 .

Concrete auction formats based on this scheme can be implemented in different ways. The most important design choices are the following:

- pricing scheme

- price update rule

- way of computing a feasible primal solution in each iteration

- stopping rule

- type of information feedback (provisional allocation, prices, etc.)

Though the effect of different pricing schemes is insufficiently studied by experiments, some conclusions can be done on the basis of the previous section. In case of discriminatory non-linear prices CE prices do always exist and a properly de- 
signed auction can be shown to converge to the efficient solution. ${ }^{9}$ Since anonymous non-linear $\mathrm{CE}$ prices do not always exist, an auction utilizing this pricing scheme is generally expected to produce less efficient results. [Park01] derives a "bid safety" condition, which makes it possible to dynamically determine the necessity of the price discrimination and to introduce it only as it is required. Linear prices usually do not support the optimal allocation in case of super- or subadditive valuations, which should in theory lead to losses in the allocation efficiency.

The price update rule is the key design feature, it differs considerably among the auction formats. In some auctions prices are increased on all overdemanded bundles, in others they are only increased on the so called "minimal overdemanded bundle sets". Often a fixed price increment is used. We will give a comparative overview of the different proposed price update rules in the section 4.

Though the primal-dual design does not require computing a feasible allocation in each iteration (in fact, the auction only needs to update prices appropriately), such allocation is usually found by maximizing the auctioneers revenue given the current bids. This "locally optimal" provisional allocation is communicated to the bidders and is declared the winning allocation when the auction terminates.

Prices are usually increased in a way to maintain the CE-condition 2 (see definition 3) over the auction runtime. In this case the auction finds CE prices as soon as no overdemanded bundles exist (all bids that are valid at the current prices belong to the provisional allocation). This condition is often used as the stopping rule.

Certainly, the primal-dual approach is not the only possibility to design an iterative combinatorial auction, but it may be the most systematical one. In the next section we describe several proposed auction formats, some of which are based on the primal-dual scheme, the others are not.

\section{Selected Auction Formats}

Most of the ICA formats have been proposed during the past few years. As already indicated in the section 2.3, the complexity of the rules makes the gametheoretic analysis much more difficult. Many researches are using simulations, laboratory experiments or field studies to investigate an auction behavior. Though some experimental results of this kind are available for individual auctions, very few comparative tests have been performed, and there is little knowledge about the preferability of specific auction formats in different settings.

9 This is shown in [Par01] and [dVSV03] for the correspondent auction respectively. The important assumptions are the myopic best-response bidding strategy and small enough bid increments. 
Figure 3 illustrates a classification of ICA. First we differentiate between the combinatorial auctions (auctions that allow package bidding) and the pseudocombinatorial auctions. One well known example of a pseudo-combinatorial auction is the Simultaneous Multiple Round (SMR) design used by the FCC to auction spectrum licenses, which works by running multiple single-item auctions simulta-

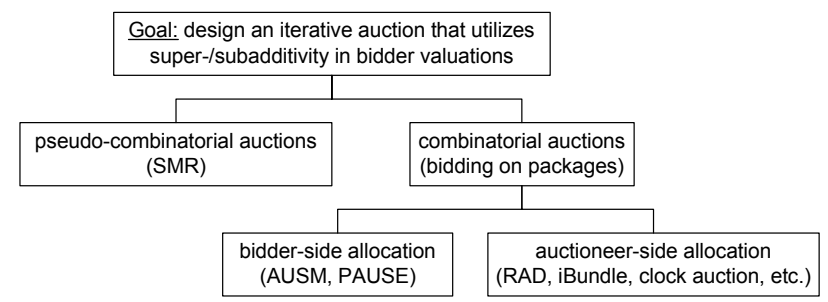

Figure 3: Iterative auction hierarchy

neously. Though the bidders are able to utilize some synergies of the simultaneous bidding, the auction suffers from the exposure problem because of the single-item bidding. The key SMR properties are summarized in the Table 5.

We further classify the combinatorial auctions into auctioneer-side allocation auctions and bidder-side allocation auctions. The bidder-side allocation auctions have been developed for small problems where bidders can cooperate in order to find a better allocation by themselves in each iteration. Two well known members of this family are the Adaptive User Selection Mechanism (AUSM) ([Ban $\left.\left.{ }^{+} 89\right]\right)$ and the Progressive Adaptive User Selection Environment (PAUSE) ([KeSt00]). Though these auctions solve the exposure problem by package bidding, they are still vulnerable to the threshold problem, require full information revelation and introduce high complexity at the bidder's side. Due to their structure these auctions do not use pricing and therefore are not the subject of this paper.

In the auctioneer-side allocation auctions the auctioneer solves the winner determination problem after the bids are collected. She then provides some kind of information feedback to support the bidders in improving their bids in the next iteration. Usually the bidder's current winning bids and the item prices are communicated. In the following subsections we give an overview of some promising auction formats belonging to this family.

\subsection{Primal-Dual Auctions with Discriminatory Pricing}

The iBundle Extend and Adjust ([Park01], [PaUn02]), Ausubel_2002 ([AuMi02]) and deVries 2003 ([Vri $\left.\left.{ }^{+} 03\right]\right)$ auctions are based on the primal-dual approach and 
utilize the discriminatory non-linear pricing scheme. ${ }^{10}$ Though the primal-dual analysis is not explicit in Ausubel 2002, the auction format clearly follows the scheme described in the section 3.2.3. The key design features are summarized in the Table 6 .

\begin{tabular}{|l|l|l|l|}
\hline & SMR & Clock (CC) & RAD \\
\hline Bid structure & items, OR & bundle, OR & bundle, OR \\
\hline Pricing scheme & anonymous linear & anonymous linear & anonymous linear \\
\hline Price used as & minimal bid price & bid price & $\begin{array}{l}\text { minimal bid price } \\
\text { (increment excluded) }\end{array}$ \\
\hline Price updates & $\begin{array}{l}\text { increase on all over- } \\
\text { dem. items }\end{array}$ & $\begin{array}{l}\text { increase on all } \\
\text { overdem. items }\end{array}$ & $\begin{array}{l}\text { compute by solving } \\
\text { several linear pro- } \\
\text { grams, can decrease }\end{array}$ \\
\hline Bid validity & $\begin{array}{l}\text { current iteration }+ \\
\text { previously winning }\end{array}$ & whole auction & $\begin{array}{l}\text { current iteration }+ \\
\text { previously winning }\end{array}$ \\
\hline Feedback & $\begin{array}{l}\text { prices, own winning } \\
\text { bids }\end{array}$ & prices & $\begin{array}{l}\text { prices, own winning } \\
\text { bids }\end{array}$ \\
\hline Stopping rule & no overdemand & $\begin{array}{l}\text { no overdemand and } \\
\text { no last iteration bid } \\
\text { is displaced }\end{array}$ & eligibility rules \\
\hline
\end{tabular}

Table 5: Iterative combinatorial auctions overview

All three auctions terminate with competitive equilibrium prices (and therefore an efficient allocation), if the bidders follow the myopic best-response bidding strategy and the bid increments are small enough. They can also generate some kind of VCG or minimal CE prices in special cases. ${ }^{11}$ The main disadvantage of these auctions is their cognitive and computational complexity, which is primarily caused by the discriminatory non-linear pricing.

\subsection{Combinatorial Clock Auction (CC)}

The Combinatorial Clock (CC) auction proposed in [Por $\left.{ }^{+} 03\right]$ can also be seen as some kind of a primal-dual auction algorithm. It utilizes anonymous linear prices which are called item clock prices. In each round bidders submit which packages they would purchase at the current prices. If overdemand holds for at least one item the price clock "ticks" for all overdemanded items (the item prices are increased by a fixed price increment), and the auction goes to the next iteration. If there is no excess demand and no excess supply, the items are allocated corre-

10 In [Par01] three different modifications of iBundle are proposed: the first with discriminatory prices, the second with anonymous prices and the third introduces the price discrimination dynamically as it is required.

11 For the definitions of the various types of prices generated by the auctions see the corresponding papers. 
sponding to the last iteration bids and the auction terminates. If there is no excess demand but there is excess supply (all active bidders on some item did not resubmit their bids in the last iteration), the auction solves the WD problem considering all bids submitted during the whole auction. If the computed allocation does not displace any active last iteration bids the auction terminates with this allocation, otherwise the prices of the respective items are increased and the auction continues. The key design features of the CC auction are summarized in the Table 5.

\begin{tabular}{|l|l|l|l|}
\hline & iBundle & Ausubel_2002 & deVries_2003 \\
\hline Bid structure & bundle, XOR & bundle, XOR & bundle, XOR \\
\hline Pricing scheme & $\begin{array}{l}\text { discr. non-linear } \\
\text { (discr. can be intro- } \\
\text { duced dynamically) }\end{array}$ & discr. non-linear & discr. non-linear \\
\hline Price used as & $\begin{array}{l}\text { minimal bid price or } \\
\text { bid price }\end{array}$ & minimal bid price & bid price \\
\hline Price updates & $\begin{array}{l}\text { increase on all over- } \\
\text { dem. bundles }\end{array}$ & $\begin{array}{l}\text { increase on all } \\
\text { overdem. bundles }\end{array}$ & $\begin{array}{l}\text { increase on mini- } \\
\text { mal overdem. set }\end{array}$ \\
\hline Bid validity & $\begin{array}{l}\text { current iteration }+ \\
\text { previously winning } \\
\text { prices, own winning } \\
\text { bids }\end{array}$ & $\begin{array}{l}\text { whole auction } \\
\text { prices, full history of } \\
\text { winning/loosing bids }\end{array}$ & prices \\
\hline Feedback & $\begin{array}{l}\text { no overdemand or no } \\
\text { new bids }\end{array}$ & $\begin{array}{l}\text { no overdemand or no } \\
\text { new bids }\end{array}$ & $\begin{array}{l}\text { no overdemand or } \\
\text { no new bids }\end{array}$ \\
\hline Stopping rule
\end{tabular}

Table 6: Iterative combinatorial auctions overview

The advantages of the $\mathrm{CC}$ auction are its cognitive, computational and communicative simplicity. However this can result in efficiency losses. One kind of inefficiency can be due to the exposure problem, since OR-bidding is used. Another kind of inefficiency can be visualized by the following example. We have 3 bidders and 3 items with the valuations $v_{l}(A B C)=5, v_{2}(A B)=2, v_{3}(C)=2$. The efficient allocation is to sell the package $(A B C)$ to the bidder 1 for the total revenue of 5. The progress of the CC auction is illustrated by table 7 .

The Clock auction would allocate the package $(A B)$ to the bidder 2 and the package $(C)$ to the bidder 3 for the total revenue of 4 , which is not efficient. This happens because the price of the package $(A B C)$ increases from 3 to 6 in the second iteration, so that the bidder 1 has no chance to reveal its real valuation of 5 .

The authors do not provide any theoretical analysis of the auction efficiency, they only claim that the auction is "simply a greedy algorithm to discover pseudo-dual upper-bound prices: The lowest prices at which everyone who submitted a bid is definitively declared a winner" ${ }^{\prime 2}$. The authors also report very good experimental efficiency results.

12 The definition of the pseudo-dual upper-bound prices is here due to [RSB82]. 


\begin{tabular}{|l|c|c|c|c|c|c|}
\hline & \multicolumn{3}{|c|}{ Prices } & \multicolumn{3}{c|}{ Active bids, prices } \\
\cline { 2 - 7 } & Item A & Item B & Item C & Bidder 1 & Bidder 2 & Bidder 3 \\
\hline Iteration 1 & 1 & 1 & 1 & $(\mathrm{ABC}, 3)$ & $(\mathrm{AB}, 2)$ & $(\mathrm{C}, 1)$ \\
\hline Iteration 2 & 2 & 2 & 2 & - & - & $(\mathrm{C}, 2)$ \\
\hline End allocation & - & - & - & - & $(\mathrm{AB}, 2)$ & $(\mathrm{C}, 2)$ \\
\hline
\end{tabular}

Table 7: Clock auction example

\subsection{Resource Allocation Design (RAD)}

The Resource Allocation Design (RAD) auction proposed in [ $\left.\mathrm{Kwa}^{+} 03\right]$ also uses anonymous linear pricing. However instead of simply increasing the prices, the auction tries to compute a set of appropriate prices compatible with the current provisional allocation by solving a number of linear programs. Since anonymous linear compatible prices do not always exist, the auction has to use approximately compatible prices: prices at which some loosing bids are still higher than the price, but the number and the deviation of such bids is minimized. Bidders have to bid more then the prices plus a fixed bid increment. The stopping rule relies on eligibility constraints which are defined similar to the eligibility constraints of the SMR auction. The key design features of the RAD auction are summarized in the Table 5 .

On the one hand, the efficiency of the RAD auction should be diminished by the anonymous linear pricing and the OR-bidding (exposure problem). However the auction gains additional flexibility, since the prices are computed dynamically. For example, the prices can be computed in a way that reduces the threshold problem. The authors provide extensive computational results proving promising efficiency results and particularly a significant gain in efficiency compared to the SMR auction.

\section{Conclusions}

The paper addresses the problem of defining market clearing prices in combinatorial auctions. As we have seen in section 4, there have been approaches to implement linear, non-linear anonymous, and even non-linear discriminatory prices. All these approaches have pros and cons. For example, allocative efficiency of nonlinear personalized prices can be traded off against bidders' perceived fairness of the prices and computational complexity. But even if non-linear prices are perceived as fair, the cognitive burden for bidders is still very high. New types of information feedback is needed to help bidders understand how much they need to improve their bids in order to win. It might also be possible to construct decision 
support tools that help bidders construct optimal bundle bids in various application domains, given information about their production schedules and workload. Extensive experimental research and field studies are needed to address these cognitive issues and develop robust combinatorial auction formats.

\section{References}

[AuMi02] L. Ausubel and P. Milgrom. Ascending auctions with package bidding. Frontiers of Theoretical Economics, 1(1), 2002.

[Bich01] M. Bichler. The Future of eMarkets: Multi-Dimensional Market Mechanisms. Cambridge University Press, Cambridge, UK, 2001.

[BiKa04] M. Bichler and J. Kalagnanam. Configurable offers and winner determination in multi-attribute auctions. European Journal of Operational Research, March, 2004.

$\left[\mathrm{Ban}^{+} 89\right]$ J.S. Banks, J. O. Ledyard, and D. Porter. Allocating uncertain and unresponsive resources: An experimental approach. RAND Journal of Economics, 20:1-25, 1989.

[BiOs02] S. Bikhchandani and J. Ostroy. The package assignment model. Journal of Economic Theory, 107(2):377-406, 2002.

[Bran03] F. Brandt. Fully private auctions in a constant number of rounds. In Financial Cryptography, Gosier, Guadeloupe, 2003.

[Cra ${ }^{+}$04] P. Crampton, Y. Shoham, and R. Steinberg, editors. Combinatorial Auctions. to appear, 2004.

[DaKa00] A. Davenport and J. Kalagnanam. Price negotiations for procurement of direct inputs. Workshop report, IMA Hot Topics Workshop: Mathematics of the Internet: EAuction and Markets, December 3-5 2000

[Vri $\left.{ }^{+} 03\right]$ S. de Vries, J. Schummer, and R. Vohra. On ascending vickrey auctions for heterogeneous objects. In FCC Combinatorial Bidding Conference 2003.

[VrVo03] S. de Vries and R. Vohra. Combinatorial auctions: A survey. INFORMS Journal of Computing, 15(3), 2003.

[ElKe02] W. Elmaghraby and P. Keskinocak. Combinatorial auctions in procurement. Technical report, Georgia Tech, 2002.

[Kal ${ }^{+}$04] J. Kalagnanam, M. Bichler, A. Davenport, and G. Hohner. Industrial procurement auctions. In P. Crampton, Y. Shoam, and R. Steinberg, editors, Combinatorial Auctions. 2004.

[Klem99] P. Klemperer. Auction theory: A guide to the literature. Journal of Economic Surveys, 13(3):227-286, 1999.

$\left[\mathrm{Kwa}^{+} 03\right]$ A. M. Kwasnica, J. O. Ledyard, D. Porter, and C. DeMartini. A new and improved design for multi-objective iterative auctions. Social Science Working Paper 1054, 2003. 
[KrRo96] V. Krishna and R.W. Rosenthal. Simultanous auctions with synergies. Games and Economic Behavior, 17:1-31, 1996.

[KeSt00] F. Kelly and R. Steinberg. A combinatorial auction with multiple winners for universal service. Management Science, 46(4):586-596, 2000.

[Levi97] J. Levin. An optimal auction for complements. Games and Economic Behavior, 18:176-192, 1997.

[Mas ${ }^{+}$95] A. Mas-Colell, M. D. Whinston, and J. R. Green. Microeconomic Theory. Oxford University Press, Oxford, UK, 1995.

[Milg87] P. R. Milgrom. Auction theory. In T. Bewley, editor, Advances in Economic Theory: Fifth World Congress. Cambridge University Press, Cambridge, UK, 1987.

[McMc87] R. McAfee and P. J. McMillan. Auctions and bidding. Journal of Economic Literature, 25:699-738, 1987.

[Nisa00] N. Nisan. Bidding and allocations in combinatorial auctions. In ACM Conference on Electronic Commerce (EC-2000), Minneapolis, MI, 2000.

[NiSe02] N. Nisan and I. Segal. The communication complexity of efficient allocation problems. Technical report, Hebrew University, 2002.

[NeWo88] G. Nemhauser and L.Wolsey. Integer and Combinatorial Optimization. John Wiley \& Sons, Chichester, 1988.

[One $\left.{ }^{+} 04\right]$ R. P. O’Neill, P. M. Sotkiewicz, B. F. Hobbs, M. H. Rothkopf, and Stewart Jr. Efficient market-clearing prices in markets with nonconvexities. European Journal of Operational Research, 2004.

[Park01] D. C. Parkes. Iterative Combinatorial Auctions: Achieving Economic and Computational Efficiency. PhD thesis, University of Pennsylvania, 2001.

[Por $\left.{ }^{+} 03\right]$ D. Porter, S. Rassenti, A. Roopnarine, V. Smith. Combinatorial auction design. Proceedings of the National Academy of Sciences of the United States of America (PNAS), 2003.

[PaSt98] C.H.; Papadimitriou and K. Steiglitz. Combinatorial Optimisation. Algorithms and Complexity. Dover Publications Inc., New York, 1998.

[PaUn02] D. C. Parkes and L. H. Ungar. An ascending-price generalized Vickrey auction. Presented at Stanford Institute for Theoretical Economics (SITE) Summer Workshop: The Economics of the Internet, 2002.

[RoPe98] M. H. Rothkopf and A. Pekec. Computationally manageable combinatorial auctions. In Maryland Auction Conference, Maryland, USA, 1998.

[Ras ${ }^{+}$82] S. J. Rassenti, V. Smith, and R. L. Bulfin. A combinatorial auction mechanism for airport time slot allocation. Bell Journal of Economics, 13:402 - 417, 1982.

[Wolf96] E. Wolfstetter. Auctions: An introduction. Journal of Economic Surveys, 10(4):367-420, 1996. References Seite 26 